\title{
Multisystem Inflammatory Syndrome in Children With COVID-19 in Mumbai, India
}

\author{
Shreepal Jain, ${ }^{1}$ Supratim Sen, ${ }^{2}$ Srinivas Lakshmivenkateshiah, ${ }^{3}$ Prashant Bobhate, ${ }^{4}$ Sumitra Venkatesh, ${ }^{1}$ \\ Soonu Udani, ${ }^{2}$ Laxmi Shobhavat, ${ }^{1}$ Parmanand Andankar, ${ }^{3}$ Tanuja Karande ${ }^{4}$ and Snehal Kulkarni ${ }^{4}$ \\ ${ }^{1}$ Bai Jerbai Wadia Hospital for Children, Mumbai; ${ }^{2}$ SRCC Children's Hospital, Mumbai; ${ }^{3}$ Jupiter Hospital, Thane; ${ }^{4}$ Kokilaben \\ Dhirubhai Ambani Hospital, Mumbai; Maharashtra, India. \\ Correspondence to: Dr Supratim Sen, Department of Pediatric Cardiology, SRCC Children 's Hospital, Mahalaxmi, Mumbai \\ 400034, Maharashtra, India.supratim80@gmail.com \\ Received: July 20, 2020; Initial review: August 04, 2020; Accepted: August 09, 2020.
}

\begin{abstract}
Objective: We describe the presentation, treatment and outcome of children with multisystem inflammatory syndrome with COVID-19 (MIS-C) in Mumbai metropolitan area in India.

Methods: This is an observational study conducted at four tertiary hospitals in Mumbai. Parameters including demographics, symptomatology, laboratory markers, medications and outcome were obtained from patient hospital records and analyzed in patients treated for MIS-C (as per WHO criteria) from 1 May, 2020 to 15 July, 2020.
\end{abstract}

Results: 23 patients (11 males) with median (range) age of 7.2 (0.8-14) years were included. COVID-19 RT-PCR or antibody was positive in $39.1 \%$ and $30.4 \%$, respectively; $34.8 \%$ had a positive contact. $65 \%$ patients presented in shock; these children

$\mathrm{M}$ ultisystem inflammatory syndrome in children (MIS-C) associated with COVID19 [1,2], also called as Pediatric inflammatory multisystem syndrome temporally associated with SARS-CoV-2 (PIMS-TS) [3], is a hyperinflammatory syndrome occurring in close temporal association with a severe acute respiratory syndrome coronavirus 2 (SARS-CoV-2) infection in children. The initial cases from India were reported in May, 2020 [4$6]$, and as the number of COVID-19 cases has grown exponentially across the country, clinicians have started identifying this new entity more frequently [7].

We describe clinical features and management in children with MIS-C from the Mumbai metropolitan area, which had a high incidence of coronavirus disease 2019 (COVID-19).

\section{METHODS}

This is the preliminary analysis of an ongoing observational study from the Division of pediatric cardiology and Division of pediatric intensive care of four had a higher age $(P=0.05)$, and significantly higher incidence of myocarditis with elevated troponin, NT pro BNP and left ventricular dysfunction, along with significant neutrophilia and lymphopenia, as compared to those without shock. Coronary artery dilation was seen in $26 \%$ patients overall. Steroids were used most commonly for treatment $(96 \%)$, usually along with intravenous immunoglobulin (IVIg) $(65 \%)$. Outcome was good with only one death.

Conclusion: Initial data on MIS-C from India is presented. Further studies and longer surveillance of patients with MIS-C are required to improve our diagnostic, treatment and surveillance criteria.

Keywords: PIMS-TS, Kawasaki disease, Myocarditis, COVID19, SARS-CoV-2.

Published Online: August 11, 2020; PII: S097475591600230

tertiary care hospitals in the Mumbai metropolitan region. Patients with MIS-C who fulfilled the WHO criteria [2] and were treated at the participating centers between 1 May, 2020 and 15 July, 2020 were included in this analysis. Other infective causes with similar presentation such as dengue shock syndrome and bacterial sepsis were excluded prior to diagnosing the patient with MIS-C. Institutional ethics committee approval was taken from all four hospitals. Data were extracted from hospital records and were entered on a Microsoft Excel spreadsheet. Variables studied included demographics, presence of positive SARS-CoV-2 antigen or antibody test or history of contact with a positive patient, clinical symptomatology, laboratory parameters, treatment given and outcome.

Based on the dominant clinical presentation, the cases were categorized into two subsets: Group 1 (MIS-C with shock), those patients requiring inotrope use and/or fluid resuscitation $>20 \mathrm{~mL} / \mathrm{kg}$; and Group 2, MIS-C without shock. Mucocutaneous features such as skin rash, non-purulent conjunctivitis, changes in lips, oral 
mucosa and extremity changes as defined by previous guidelines of Kawasaki disease (KD) [8] and MIS-C [2] were noted. Shock was defined as hypotension with poor peripheral perfusion requiring inotropic support and/or fluid resuscitation $>20 \mathrm{~mL} / \mathrm{kg}$. Specific laboratory markers described for MIS-C were measured as per the treating physician's discretion and institutional protocols. This included total and differential white blood cell count, platelet count, acute phase reactants (C-reactive protein, ferritin, D-dimer, interleukin-6), renal and liver function tests and cardiac biomarkers (troponin, CPK-MB, Nterminal pro BNP). Laboratory parameters were labelled as elevated or depressed in relation to the age-specific normal ranges.

Echocardiography was used to assess ventricular dimensions, myocardial dysfunction and ectasia or aneurysm of the coronary arteries. All echocardiograms were done by the consultant pediatric cardiologists at the respective centers. Presence of arrhythmias and ischemia was assessed on electrocardiogram. Chest CT was not routinely done. Clinical myocarditis was defined as cardiac dysfunction with left ventricular ejection fraction (LVEF) $<50 \%$ on echocardiography with elevated cardiac biomarkers. Patients presenting with shock with left ventricular (LV) dysfunction on echocardiography were classified primarily as cardiogenic shock. Patients with warm shock requiring inotropic support in spite of having normal left ventricular function were classified as vasoplegic shock. The coronary artery diameters were measured as per standard criteria [8] and indexed with $Z$ scores [9]. Coronary $Z$ scores of greater than 2.5 were considered as dilated [8].

SARS-CoV-2 infection was diagnosed by nasopharyngeal swab real-time reverse transcription-polymerase chain reaction (RT-PCR) and/or rapid antibody test for SARS-CoV-2 (Vitros Anti Sars Cov IgG antibody kit, Ortho Clinical Diagnostics) as recommended by Indian Council for Medical Research. Additionally, history of contact with a COVID19 positive patient was also considered positive as per the WHO criteria. Outcome was classified as discharged or death. Long term follow-up of these patients is ongoing.

Statistical analyses: Statistical analysis was performed using SPSS v 26 (IBM, USA). Chi-square test was used to compare categorical variables, student-t test was used to compare normally distributed data and Mann Whitney U test was used to compare data which was not normally distributed.

\section{RESULTS}

A total of 23 patients (11 males) with MIS-C were treated during the study period. Demographics and clinical presentation are detailed in Table I and laboratory findings and treatment are shown in Table II. Patients presenting with shock (group 1) were older and had significantly higher neutrophil count, lower lymphocyte counts, higher serum ferritin, NT pro BNP and troponin levels as compared to group 2. Of the patients in group 1 $(n=15), 8(53 \%)$ had LV dysfunction with cardiogenic shock; those with normal LV function who also presented in shock possibly had vasoplegic shock with the generalized hyperinflammatory state. Clinical myocarditis was diagnosed in 15 patients (65\%) who had LV dysfunction and/or elevated cardiac biomarkers and coronary involvement was seen in $26 \%$ of the patients.

One six-year old girl with positive COVID contact with history of fever and loose stools, presented to the casualty in shock, died within two hours of presentation with pulmonary hemorrhage despite all management efforts (Shock management, steroids, IVIg, invasive ventilation, antibiotics). Her echocardiogram showed moderate LV systolic dysfunction (LVEF 37\%). RT-PCR sent at admission was negative, and antibody testing could not be done.

\section{DISCUSSION}

MIS-C is a rare disorder, affecting only $0.6 \%$ of patients $<21$ years of age infected with SARS Cov-2 $[10,11]$ and

Table I Demographic and Clinical Parameters in Children With Multisystem Inflammatory Syndrome in Children With COVID-19 (MIS-C)

\begin{tabular}{|c|c|c|c|}
\hline Characteristics & $\begin{array}{l}\text { Total cases } \\
n=23\end{array}$ & $\begin{array}{l}\text { Group } 1= \\
\text { Shock, } \\
n=15\end{array}$ & $\begin{array}{r}\text { Group } 2= \\
\text { No shock, } \\
n=8\end{array}$ \\
\hline${ }^{\wedge} \operatorname{Age}(y)^{*}$ & $7.2(5.7-9.4)$ & $7.8(6-10.1)$ & $5.2(1.25-9.7)$ \\
\hline Females & 12 & $9(60)$ & $3(37.5)$ \\
\hline $\mathrm{H} / \mathrm{o}$ contact & $8(34.8)$ & $5(33.3)$ & $2(25)$ \\
\hline RT-PCR positive & $9(39.1)$ & $5(33.3)$ & $4(50)$ \\
\hline Antibody positive & $7(30.4)$ & $4(26.6)$ & $3(37.5)$ \\
\hline \multicolumn{4}{|l|}{ Symptoms } \\
\hline Fever duration $(\mathrm{d})^{\#}$ & $5.2(1.8)$ & $5.5(0.6)$ & $4.75(1.5)$ \\
\hline Pain in abdomen & $12(52.1)$ & $8(53.3)$ & $4(50)$ \\
\hline Diarrhea/vomiting & $15(69.5)$ & $9(60)$ & $6(75)$ \\
\hline Breathlessness & $11(47.8)$ & $9(60)$ & $2(25)$ \\
\hline Rash & $14(65.2)$ & $9(60)$ & $5(62.5)$ \\
\hline Conjunctivitis & $11(52.1)$ & $7(46.7)$ & $4(50)$ \\
\hline Oral cavity changes & $4(21.7)$ & $2(13.3)$ & $2(25)$ \\
\hline Limb changes & $3(13.0)$ & $2(13.3)$ & $1(12.5)$ \\
\hline $\mathrm{SpO}_{2}(\%)^{\#}$ & $95.9(7.1)$ & $95.8(8.7)$ & $96.1(3.4)$ \\
\hline
\end{tabular}


Table II Biochemical and Echocardiographic Parameters and Management in Children With MIS-C

\begin{tabular}{|c|c|c|c|c|}
\hline Characteristics & Total $(n=23)$ & Group I $(n=15)$ & Group II $(n=8)$ & Pvalue \\
\hline \multicolumn{5}{|l|}{ Investigations } \\
\hline Total leucocyte count $\left(\mathrm{X} 10^{9}\right)$, mean (SD) & $15.0(10.2)$ & $16.2(1.0)$ & $11.3(8.1)$ & 0.26 \\
\hline Neutrophils percent, mean (SD) & $80(11)$ & $83(10)$ & $67.1(6.7)$ & 0.007 \\
\hline Lymphocyte percent, mean (SD) & $14.3(9.1)$ & $11.4(8.0)$ & $20.5(8.5)$ & 0.02 \\
\hline Hemoglobin (gm/dL), mean (SD) & $10.4(2.2)$ & $10.1(2.6)$ & $11.2(1.1)$ & 0.18 \\
\hline Platelet $\left(\mathrm{X} 10^{9}\right)$, mean $(\mathrm{SD})$ & $236.8(155.9)$ & $185.1(133.8)$ & $323.6(165.2)$ & 0.06 \\
\hline $\begin{array}{l}\text { Serum glutamic pyruvic transaminase } \\
(\mathrm{U} / \mathrm{L}) \text {, median }(\mathrm{IQR})\end{array}$ & $48(23-89.5)$ & $72(38-209)$ & $26(14-35)$ & 0.007 \\
\hline Creatinine (mg/dL), median (IQR) & $0.47(0.35-0.6)$ & $0.5(0.3-0.7)$ & $0.37(0.27-0.49)$ & 0.17 \\
\hline Creactive protein (mg/L), mean (SD) & $96.6(67.03)$ & $107(72)$ & $87(57)$ & 0.65 \\
\hline Serum ferritin (ng/mL), median (IQR) & $596.8(282.2-1473.5)$ & $875(422-2338)$ & $319(85-515)$ & 0.01 \\
\hline D-dimer (ng/mL), median (IQR) & $4090(1824.9-9958.7)$ & $3160(1827-10330)$ & $5609(1770-13765)$ & 0.78 \\
\hline Interleukin $6(\mathrm{pg} / \mathrm{mL})$, median (IQR) & $230.2(95.5-498.7)$ & $351(172-635)$ & $95.5(33-359)$ & 0.12 \\
\hline NT-Pro BNP (pg/mL), median (IQR) & $410(205.5-21277)$ & 35000 & $205(101-382)$ & 0.05 \\
\hline Troponin (ng/mL), median (IQR) & $33.4(5.7-185)$ & $79.4(10.8-360)$ & $0.1(0.01-0.1)$ & 0.007 \\
\hline \multicolumn{5}{|l|}{ Echocardiographic features } \\
\hline LV systolic dysfunction, $n(\%)$ & $8(34.8)$ & $8(53.3)$ & 0 & 0.01 \\
\hline Coronary dilation, $n(\%)$ & $6(26)$ & $3(20)$ & $3(37.5)$ & 0.3 \\
\hline \multicolumn{5}{|l|}{ Treatment } \\
\hline Mechanical ventilation, $n(\%)$ & $9(39.1)$ & $8(53.3)$ & $1(12.5)$ & 0.05 \\
\hline IVIG, $n(\%)$ & $15(65.2)$ & $12(80)$ & $3(37.5)$ & 0.04 \\
\hline Steroids, $n(\%)$ & $22(95.6)$ & $15(100)$ & $7(87.5)$ & 0.16 \\
\hline Tocilizumab/infliximab, $n(\%)$ & $3(13.0)$ & $3(20)$ & 0 & 0.17 \\
\hline
\end{tabular}

MIS-C: Multi-system inflammatory syndrome in children; Left ventricular (LV) systolic dysfunction: Ejection fraction $<50 \%$ as measured by $M$ Mode echocardiography in parasternal long axis view; Coronary involvement: $Z$ score $>2.5$ of either the left main coronary artery, left anterior descending coronary artery or the right coronary artery. NT Pro BNP: $N$ terminal pro brain natriuretic peptide.

there are limitations in its recognition and diagnosis [11]. Within India, Mumbai was one of the early epicenters of the epidemic and we believe this is one of the reasons that this region has witnessed an early clustering of MIS-C. Testing guidelines with COVID-19 RT-PCR have now been streamlined in Mumbai and most parts of India. However, the antibody test reports are still not standardized, and IgM and IgG levels are not routinely reported. Hence, the requirement of either antigen or antibody positivity, or positivity in an immediate contact, is a potential problem in any COVID-related diagnosis, including MIS-C. In the absence of standardized uniform international diagnostic guidelines, there is high probability that clinicians are either missing the milder cases [11] or even over-diagnosing similar presentations of KD or toxic shock syndrome [5] as MIS-C.

Whitaker, et al. [3] have proposed three clinical patterns of PIMS-TS presentation viz, those with shock and cardiac involvement, those with fever and elevated inflammatory markers without features of $\mathrm{KD}$, and those who fulfilled diagnostic criteria for $\mathrm{KD}$. In our series, while only one patient fulfilled diagnostic criteria for classical KD [8], there was significant clinical overlap of several patients having few mucocutaneous features of $\mathrm{KD}$, raised inflammatory markers, and presenting with shock. The most important clinical factor in our patients affecting treatment and outcomes was shock. As expected, the levels of NT pro-BNP and troponin were significantly elevated in the group with shock, and inotropic and ventilatory requirements were more. Additionally, neutrophilia, lymphopenia, elevated serum ferritin and liver enzymes were significant laboratory parameters observed in patients with shock. Use of IV immunoglobulin (IVIG) was significantly more in this group of our patients, possibly because they were sicker at admission. Shock, myocarditis and LV dysfunction were all more common in older children in our series.

Dufort, et al. [10] reported KD/KD like illness in 36\%, 


\section{WHAT THIS STUDY ADDS}

- The clinical presentation, laboratory findings, response to treatment and outcome in children affected with MIS-C are reported.

myocarditis in $53 \%$, shock in $10 \%$ and coronary aneurysms in $9 \%$ of their cohort of children with MIS-C from New York. In a recent study from Chennai, India, Dhanalakshmi, et al. reported hypotension requiring vasoactive medications in $57 \%$ of patients presenting with PIMS-TS, and coronary artery changes in 16\% [7]. Previous authors have observed coronary involvement in all clinical groups of MIS-C, regardless of laboratory markers and whether diagnostic criteria of $\mathrm{KD}$ were met [3]. This observation is consistent with the present series as coronary involvement did not differ statistically with shock or age at presentation in our patients. This implies that all patients with MIS-C would need serial echocardiographic surveillance for coronary and myocardial involvement in the acute and convalescent phase of illness, even if the initial echocardiogram was normal $[3,12]$, at least until definite guidelines for long term cardiac follow-up after MIS-C become available.

In classifying patients with MIS-C with Kawasaki-like illness, clinicians need to carefully differentiate this from classical KD in patients from COVID-19 endemic areas [6]. There is significant epidemiological evidence that MIS-C is distinct from $\mathrm{KD}$. Children with MIS-C are older and sicker a compared with those of classical KD [3,10,12]. Feldstein, et al. [12] have observed 50\% MIS-C patients presenting with cardiovascular shock leading to vasopressor or inotropic support as compared to only $5 \%$ of children with KD in the United States. Similarly, in our series, the median age of patients was 7.2 years, which is older than the age of presentation for $\mathrm{KD}$.

There is a 2-4 week lag period for MIS-C presentation post COVID-19 infection, and we should expect to see more patients from across India in the coming weeks, based on present infection trends. In the US MIS-C series, IVIG (77\%) and systemic glucocorticoids (49\%) were used in most patients [12]. In the UK series, $71 \%$ received IVIG and $64 \%$ corticosteroids. Three patients received anakinra and eight received infliximab. Inotropic support was required in $47 \%$ [3]. In our series, $96 \%$ of the patients received steroids and $65 \%$ IVIg, and $65 \%$ required inotropic support. Biologicals such as tocilizumab/infliximab were used in $13 \%$. The relatively lower usage of IVIg can be attributed to the high cost of this treatment, which unfortunately is often a deciding factor for treatment decisions in our population.
Based on our small numbers, we do not believe that at present, levels of acute phase reactants can reliably predict the subsequent clinical course of the child. The cardiac biomarkers (NT pro BNP, Troponin and CPK-MB) are of course indicative of myocarditis and can be used to predict clinical deterioration and shock. Generally, the short-term outcomes of MIS-C have been promising. Mortality in our series was $4.3 \%$, which is comparable to international studies [11].

Our study is an ongoing analysis of hospital data. We were rigid in our case selection to include only those patients who themselves or whose immediate family contacts had confirmed SARS-CoV-2 antigen or seropositivity. The main prerequisite for these diagnostic criteria is universal and free availability of testing, which is often not the case. Hence, we may have missed mild cases or reallocated them to a diagnosis of KD due to this testing criterion. Additionally, we have only reported echocardiography and coronary findings at presentation. As we know from the KD experience, serial surveillance for coronary involvement with follow up echocardiography is essential to understand the mid-term and longterm sequelae of MIS-C in our patient population.

Our preliminary data is expected to add to the meagre data on this condition from India, and assist clinicians in identifying and managing MIS-C. Further studies and longer surveillance of patients diagnosed with MIS-C is required to improve our diagnostic, treatment and surveillance criteria.

Acknowledgements: The following pediatricians, pediatric intensivists and pediatric cardiologists who were involved in the clinical care of the patients studied: Dr Jayashree Mishra, Dr Amish Vora, Dr Aoyan Sengupta, Dr Bharat Parmar, Dr Indu Khosla, Dr Shashank Parekhji, Dr Sudhir Sane and Dr Priya Pradhan. We would also like to thank Dr Tanu Singhal for her expert advice in the preparation of the manuscript.

Ethics clearance: Institutional Ethics Committee (IEC) BJ Wadia Hospital for Children; No. IEC-BJWHC/66/2020, dated July 18, 2020. SRCC Children's Hospital Ethics Committee; No R202010, dated June 30, 2020. Jupiter Hospital IEC; dated July 8, 2020. KDAH Ethics Committee; ECR/141/Inst/MH/2013/RR19, dated June 6, 2020.

Contributors: SK, SS, SL, SV, TK, PA, LS, SU: contribution to the conception and design of the work, preparation and finalization of the draft; SL, SJ, PB, SS: contributions to the acquisition, analysis, and interpretation of data for the work; SK, 
SS, PB, SU: critical revision. All authors approved the final version of the manuscript.

Funding: None; Competing interests: None stated.

\section{REFERENCES}

1. Multisystem Inflammatory Syndrome in Children (MISC) Associated with Coronavirus Disease 2019. Centers for Disease Control and Prevention. Emergency preparedness and response: Health alert network. May 14, 2020. Available from: https://emergency.cdc.gov/han/2020/ han00432.asp. Accessed July 15, 2020.

2. Multisystem inflammatory syndrome in children and adolescents with COVID-19. Scientific brief: World Health Organisation. 15 May 2020. Available from: https: $/ / w w w . w h o . i n t / p u b l i c a t i o n s-d e t a i l / m u l t i s y s t e m i n f l a m-$ matory-syndrome-in-children-andadolescents-with-covid19. Accessed July 15, 2020.

3. Whittaker E, Bamford A, Kenny J, Kaforou M, Jones CE, Shah $\mathrm{P}$, et al. Clinical characteristics of 58 children with a pediatric inflammatory multisystem syndrome temporally associated with SARS-CoV-2. JAMA. 2020; e2010369. [E-pub ahead for print].

4. Balasubramanian S, Nagendran TM, Ramachandran B, Ramanan AV. Hyper-inflammatory syndrome in a child with COVID-19 treated successfully with intravenous immunoglobulin and tocilizumab. Indian Pediatr. 2020; S097475591600180. [published online ahead of print].

5. Bhat CS, Gupta L, Balasubramanian S, Singh S, Ramanan AV. Hyperinflammatory Syndrome in Children Associated with COVID-19: Need for Awareness. Indian Pediatrics. 2020; S097475591600208. [published online ahead of print]
6. Acharyya BC, Acharyya S, Das D. Novel coronavirus mimicking Kawasaki disease in an infant [published online ahead of print, 2020 May 22]. Indian Pediatr. 2020;S097475591600184.

7. Dhanalakshmi K, Venkataraman A, Balasubramanian S, Madhusudan M, Amperayani S, Putilibai S et al. Epidemiological and clinical profile of pediatric inflammatory multisystem syndrome - temporally associated with SARSCoV-2 (PIMS-TS) in Indian children. Indian Pediatr. 2020; S097475591600220. [published online ahead of print]

8. McCrindle BW, Rowley AH, Newburger JW, Burns JC, Bolger AF, Gewitz M, et al. Diagnosis, Treatment, and Long-term Management of Kawasaki Disease: A Scientific Statement for Health Professionals from the American Heart Association. Circulation. 2017;135: e927-99.

9. McCrindle BW, Li JS, Minich LL, Colan SD, Atz AM, Takahashi M, et al; Pediatric Heart Network Investigators. Coronary artery involvement in children with Kawasaki disease: risk factors from analysis of serial normalized measurements. Circulation. 2007;116:174-9.

10. Dufort EM, Koumans EH, Chow EJ, Rosenthal EM, Muse A, Rowlands J, et al. Multisystem inflammatory syndrome in children in New York State. N Engl J Med. 2020;383: 347-58.

11. Levin M. Childhood multisystem inflammatory syndrome- A new challenge in the pandemic. N Engl J Med. 2020. 10.1056/NEJMe2023158 (Published online ahead of print).

12. Feldstein LR, Rose EB, Horwitz SM, Collins JP, Newhams MM, Son MBF, et al. Multisystem inflammatory syndrome in U.S. children and adolescents. N Engl J Med. 2020; 383:334-6. 